\title{
Formación del arquitecto: La incidencia del proyecto de título en el campo laboral
}

doi: 10.33264/rpa.202101-14

\author{
Domingo Andrés Sarmiento Lara
}

Guillermo Wladimir Caro Pinto

\section{Resumen}

Este artículo desarrolla un análisis reflexivo sobre el aporte que genera el proceso de titulación tradicional (que es utilizado por la mayoría de las escuelas del país) basado en el desarrollo de un anteproyecto ficticio como parte de la formación del arquitecto y su relación con el ejercicio profesional. Estudios previos infieren sobre la distancia entre el proceso de título y el mercado laboral del arquitecto. El objetivo es describir la incidencia, en el ejercicio profesional, que tiene el proceso de titulación vigente en la carrera de arquitectura de una universidad privada de la Región Metropolitana. Para este estudio, se trabajó desde una metodología mixta (CUALI+Cuanti), con un enfoque exploratorio/fenomenológico, en el que se aplicaron entrevistas semi estructuradas y encuestas a 12 alumnos en proceso de titulación y 20 arquitectos egresados de la misma institución, con 3 a 7 años de ejercicio profesional. Los resultados evidencian que la institución debería contar con nuevos métodos de titulación que acerquen al futuro arquitecto a las problemáticas reales y actuales del campo laboral. En conclusión, la titulación a través de un anteproyecto ficticio no incide significativamente y no se relaciona con el ejercicio de la profesión. Se infiere entonces que las escuelas de arquitectura deberían entregar nuevas y mejores opciones de titulación, a partir de los problemas concretos y reales de la sociedad, vinculados al mercado laboral actual de la disciplina.

Palabras clave: Arquitectura, Proyecto de Título, Proceso de Titulación, Egresados, Campo Laboral.

\section{Abstract}

This paper develop a reflexive analysis about the contribution generated by the traditional degree process (which is used by most schools of the country) based on the development of a fictitious blueprint as part of the architect's formation and its relationship with professional practice. Previous studies infer about the distance between the title process and the architect's labor market. The objective is to describe the incidence, in professional practice, of the current qualification process in the architecture career of a private university in the Metropolitan Region. For this study, we worked from a mixed methodology (CUALI + Cuanti), with an exploratory / phenomenological approach, in which semi-structured interviews and surveys are applied to 12 students in the process of qualification and 20 architects graduated from the same institution, with 3 to 7 years of professional practice. The results show that the institution should have new methods of certification that bring the future architect to the real and current problems of the labor field. In conclusion, the degree through a fictitious blueprint does not significantly affect and does not relate to the 
exercise of the profession. It is inferred then that the schools of architecture should deliver new and better options of certification, from the concrete and real problems of society linked to the current labor market of the discipline.

Keywords: architecture, degree project, degree process, graduates, labor market

\section{Introducción}

El mundo cambia cada vez con más celeridad y apremio. Actualmente el rol del arquitecto en nuestra sociedad no está del todo claro, ya que éste ha difuminado los dogmas entorno a su quehacer, buscando abrirse paso en un mercado cada vez más difícil. El número de arquitectos que se titula año a año de las diferentes Escuelas de Arquitectura en Chile supera a los 1243 nuevos profesionales (Ministerio de Educación, 2018), éstos copan con creces el mercado disponible para el quehacer “tradicional" de la profesión. En la actualidad el arquitecto se desempeña en variados escenarios insospechados hace algunos años. El mercado nos exige como gremio re inventarnos y aprovechar aquello en nuestra formación, lo que nos permite enfrentarnos a diferentes desafíos con una gran calidad en el desempeño. Es aquí donde surge el problema de estudio, somos nosotros los arquitectos los que, al vernos enfrentados a un mercado laboral hostil, debemos ser capaces de reinventarnos, reformularnos en el momento en que no encontramos el espacio que se nos prometió tácitamente al ingresar a la universidad. Aquí es cuando cuestionamos con mayor fuerza lo desarrollado en la academia en los años previos, sobre todo en la fase final de nuestra formación donde nos vemos enfrentados al proceso de titulación.

Vemos cómo en algunas ocasiones abordamos forzosamente un proyecto académico muy distante de la realidad en la que nos desempeñamos; donde el esfuerzo, el dinero, el tiempo y la dedicación de, en el mejor de los casos, año y medio de proceso, nos parece un desperdicio, basados en el hecho de que nuestras expectativas apuntaban a que este trabajo final nos permitiera abrirnos camino al campo laboral, que es el lugar donde soñamos poder dar rienda suelta a nuestros talentos, capacidades y competencias profesionales.

Coincidentemente, González (2017) establece que para un egresado, el rápido ingreso al mercado laboral depende principalmente de la preparación que recibe en su paso por la Universidad. Por ende, la formación del nuevo profesional debe estar orientada en este sentido. 
De acuerdo a lo anterior, como arquitectos, sentimos que el mercado está exigiendo a la academia actualizar distintos aspectos de la formación, a modo de ejemplo, La escuela de Arquitectura de la Universidad en estudio, solía tener un $88 \%$ de empleabilidad, sin embargo, el año 2017 este índice bajó a 76\%. (Fuente: mifuturo.cl).

Salvo honrosas excepciones, la academia se mantiene fiel de manera dogmática a una forma de hacer título que debe tener una antigüedad de al menos 50 años, quizás más. Por lo tanto, se infiere que el momento que marca un hito, donde un futuro arquitecto deja de ser estudiante y pasa a ser un profesional, se mantiene fiel a un mercado y sociedad que ya no existen, más allá del romanticismo y melancolía de quienes están a cargo de las diferentes instituciones de educación superior. Lo que buscamos en este trabajo es estudiar, contrastar, verificar y analizar esto. Quizás somos nosotros los equivocados y los procesos de titulación actuales están siendo útiles en la inserción laboral de nuestros jóvenes arquitectos. Ahora bien, si esto no se está cumpliendo y la sensación de separación de la academia con el mundo real existe, este trabajo podría llegar a ser un documento clave que impulse los cambios que nuestra disciplina requiere. Más allá de las creencias personales que cada uno pueda tener, es necesario contar con datos duros que corroboren o descarten las diferentes creencias al respecto y por cierto sirvan para tomar decisiones debidamente informadas.

Por lo tanto, hoy por hoy, no se ha determinado si las expectativas de los y las estudiantes en su última fase de egreso y previa a la titulación de la carrera de arquitectura en una Universidad Privada de Santiago, están en sintonía con las destrezas y habilidades que el campo laboral requiere.

\section{Metodología}

\section{a)Paradigma y Diseño:}

Siguiendo un enfoque exploratorio y fenomenológico, la investigación busca definir la concepción del mundo con exigencia propia independiente de quien lo estudia y comprender los sentidos de los actores involucrados. De este modo, el enfoque es mixto CUALI-cuanti, permite incorporar al diseño Cuali instrumentos cuantitativos, para nutrir la primera, generando una triangulación metodológica de las categorías en estudio.

Así, el estudio es descriptivo de corte interpretativo, con una secuencia de gabinetes (diseño y validación) y Terreno (donde se aplican datos), puesto que su directriz es determinar si las variables a estudiar están relacionadas positiva o negativamente y, al mismo tiempo, entablar comparaciones para analizar la problemática de interés en estudiantes en proceso de titulación y egresados. Concorde a lo dicho, es exploratorio 
fenomenológico, a levantar la información entre junio y agosto del año 2018.

El instrumento fue diseñado para obtener un amplio espectro de opiniones. Una serie de preguntas que revelan de parte del estudiante en proceso de titulación sus expectativas y opiniones, para luego plantear las mismas preguntas a profesionales que pasaron por el proceso hace años y que ahora en distancia pueden opinar racional y pausadamente sobre la incidencia de este proceso en su actual vida laboral. Se cuidó de mantener una relación cerrada entre el proceso de título y el desempeño laboral de los mismos.

b)Población sobre la que se efectuará el estudio:

La población sobre la cual se efectuará el estudio mixto (CUALI y cuanti) corresponden a 12 alumnos que estén en proceso de titulación, pertenecientes a una escuela de arquitectura de una universidad privada en Santiago de Chile y 20 titulados de la misma institución, con 3 a 7 años de egreso. De esa forma, se podría asegurar que estuvieran insertos en el campo laboral.

Para los sujetos en estudio, no hubo filtros de edad, sexo, ni nivel socio económico, solamente se consideró su condición de estudiante y titulado de la carrera de arquitectura, en la universidad de estudio.

\section{c)Entorno:}

Para realizar la investigación, se solicitó previamente al director de escuela de arquitectura la autorización para realizar el estudio y junto a esto, una base de datos con los correos electrónicos de los egresados de la escuela de arquitectura de hasta 7 años de ejercicio profesional. Para contactar a los alumnos y alumnas en proceso de titulación, tuvimos que solicitar a los profesores de los talleres de titulación, los correos de sus alumnos. Las preguntas de desarrollo y encuestas, fueron enviadas por correo electrónico mediante formulario de Google, sin haber interacción persona a persona. No obstante, algunos entrevistados nos solicitaron hablar mediante teléfono, para que les explicáramos en qué consistía el correo enviado, a quienes se les explicó y accedieron a contestar la encuesta.

\section{d)Intervenciones:}

Respecto a las intervenciones, las técnicas a utilizar son encuestas tipo Likert y entrevistas semiestructuradas (Canales, 2006). La primera, corresponde a "una secuencia de enunciados que logran evaluar en qué grado una persona está de acuerdo o en desacuerdo con ellos" (Delgado y Gutiérrez, 1999, p.380), ésta no discrimina sexo, edad o nivel socioeconómico y consta de 15 preguntas. Se utilizará una escala de apreciación, categorizada en cuatro niveles (muy de acuerdo, de acuerdo, en desacuerdo y muy en desacuerdo), con sentencias afirmativas. 
La segunda, en cambio, es un formato flexible de preguntas, adquiriendo sentido el intercambio de opiniones entre entrevistador y entrevistados (Canales, 2006).

La aplicación de la encuesta y entrevista, se realizó mediante formulario de Google. Se enviaron al correo electrónico de los sujetos de estudio, según la base de datos entregada por la dirección de escuela de la universidad. Se consideraron como criterios de calidad de la información: validez y confiabilidad (Flick, 2004).

\section{e)Rigor Científico:}

Tanto las preguntas semi estructuradas, auto administradas, como las encuestas tipo Likert, aplicadas a todos los sujetos de estudio, fueron validadas por 5 expertos, todos con grado de Magíster o Doctorados, entre los que se encontraban: 3 docentes arquitectos de la carrera de arquitectura, con grado de Magíster, 1 docente no arquitecto, con grado de Doctor y 1 docente universitario con grado de Magíster en Psicología Clínica.

Se envió una primera propuesta de las preguntas, en formato Word, vía correo electrónico, en donde se pide aprobar o desaprobar cada pregunta con un sí y un no. A cada pregunta se le deja un espacio para que el validador agregue sus observaciones. Todos respondieron con observaciones que fueron acogidas, realizando una segunda versión de las preguntas, las que fueron nuevamente revisadas y aprobadas, para posteriormente ser aplicadas.

\section{f)Aspectos éticos:}

Para la presente investigación, se usó formulario Google, el cual fue enviado a los correos obtenidos de la base de datos entregada por la dirección de la escuela de arquitectura, que contenía información de egresados con 2 a 7 años de ejercicio profesional (al momento de recibir las respuestas, los años de egreso difirieron a lo entregado en la planilla, por lo que se modificaron los años para los egresados cambiando a 3 y 7 años). Posteriormente, se solicitaron a la dirección los correos de los profesores titulares de los talleres de titulación, a quienes se les pidió vía correo electrónico los datos de sus alumnos y alumnas, para hacerles llegar las encuestas.

Para este proceso, se le pidió al director de escuela firmar una carta de apoyo, en donde se comprometió a entregar la base de datos antes mencionada.

Cada formulario comienza con una breve explicación de la investigación, y objetivo de las preguntas, pasando posteriormente a la pregunta inicial, la que pide al entrevistado participar voluntariamente de esta investigación, solamente marcando las respuestas Sí o NO. El participante sólo podía ver y responder las preguntas una vez que declarara su interés en participar al hacer clic en la opción correspondiente. 
Si se hacía clic en la opción negativa, el formulario se cerraba automáticamente.

Cabe señalar que cada participante se indica que su identidad se mantendrá en completo anonimato, por lo que a cada uno se le asignó un código para representado en esta investigación:

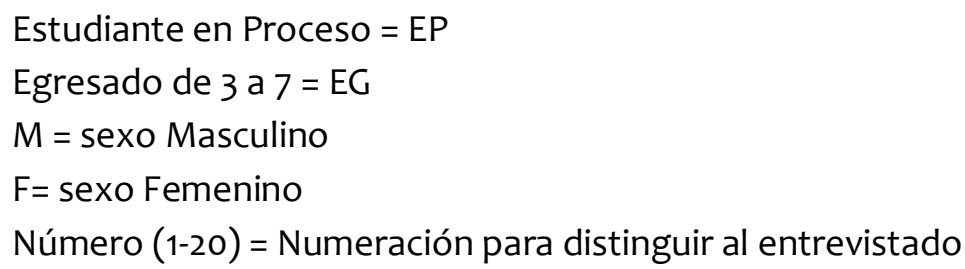

g)Plan de análisis de los datos:

Finalmente, el plan de análisis de los datos converge en una mixtura Cualitativacuantitativa, entrevistas semi estructuradas auto administradas y encuestas tipo Likert.

El análisis de datos se planteó de la siguiente manera: al recibir las entrevistas contestadas, fueron sometidas a una primera revisión, en la cual nos comunicamos con el colaborador en la medida que algo en sus respuestas no quedase absolutamente claro o debidamente expresado. Esto con el fin de asegurar que el documento recibido comunicara por sí mismo lo que él deseaba expresar, evitando así interpretaciones de parte nuestra o de terceros.

Terminada esta primera etapa, pasamos a una revisión final de los textos recibidos, donde se descartaron aquellos textos que estuviesen demasiado cargados hacia cualquiera de los extremos, ya sea este positivo o negativo, del mismo modo se eliminaron aquellos que no se expresaban en términos académicos o profesionales. Junto a esto se realizó de la selección de los textos a trabajar y la revisión de puntajes del instrumento Likert.

Contando con las textualidades y los puntajes de las encuestas, luego se separaron los diferentes grupos y se realizó el proceso de comparaciones de opiniones y puntaje, quedando así la información separada en los siguientes grupos:

-Alumnos en proceso de titulación Universidad en estudio (12) -Egresados Universidad en estudio con 3 a 7 años de ejercicio profesional (20)

La separación de los grupos tiene por motivo el lograr las comparaciones en los diferentes niveles. Primero fueron comparadas las opiniones de los diferentes 
colaboradores de cada grupo entre sí. Posteriormente, los resultados de cada grupo fueron contrastados entre ellos, alumnos en proceso de título y profesionales con 3 a 7 años de egreso de la misma escuela de arquitectura. Luego revisamos el contraste de las expectativas y percepción de la incidencia del proceso de título entre los diferentes grupos.

Finalmente contamos con un compilado del mundo académico que se compara a sí mismo y que a su vez se compara con el mundo profesional en torno al proyecto de título, su desarrollo, su pertinencia y la incidencia de este en las exigencias del mundo laboral para el arquitecto.

\section{Resultados y Análisis}

A partir de los datos levantados, y la interpretación de la presente matriz de codificación selectiva, podemos deducir que hoy por hoy el mundo educativo entiende que la empleabilidad se ha convertido en uno de los pilares de los nuevos modelos educativos, basados en competencias, trabajando como centro de gravedad el aprendizaje de los estudiantes (Michavila, 2018). Lo anterior nos lleva a preguntamos: ¿para qué sociedad y/o mercado laboral estamos formando nuestros futuros arquitectos? (González 2017). ¿Las competencias que poseen nuestros egresados son coincidentes con las exigencias de los empleadores? ¿Nuestro planteamiento ha logrado desprenderse de la formación dogmática centrada en estudios de órdenes, composición, dejado de lado problemas técnicos medulares del ejercicio profesional? (Quesada, 2016) ¿Logramos entregar al mercado arquitectos proactivos, capaces de responder a las complejas realidades de nuestra sociedad actual? (Cabrera, 2018). Estas preguntas nos llevan a poner en cuestionamiento el actual proceso de titulación de la universidad en estudio, en cuanto si es capaz de ser el articulador entre la evaluación de las competencias profesionales de nuestros estudiantes y la inserción al campo laboral, o al menos preguntarnos si realmente es un aporte en su formación profesional.

Mientras nos hacemos estas preguntas, los estudiantes y egresados establecen que no existe una vinculación directa entre el proceso de titulación y el campo laboral (EPM-10), que no sienten que tengan apoyo por parte de la institución y profesores guías para realizar esta vinculación (EP-M-12). Tanto las experiencias laborales que los estudiantes han adquirido, como las prácticas profesionales realizadas, les han permitido ver que existe una brecha entre lo que les enseñan en la universidad y el ejercicio profesional (EP-F-11), expresando, en algunos casos, que la formación académica debería acercarse al mundo real (EG-3F-8); otros incluso consideran la universidad como una utopía (EG-4F-6). 
Ahora, si consideramos que los índices de empleabilidad de la universidad han caído constantemente durante los últimos años, podrían tener razón. La preocupación de ellos radica en que poseen el concepto de "adquirir educación de calidad para obtener trabajo" (González 2017). A pesar de esto, no todos los datos recogidos son negativos.

Los estudiantes y egresados que participaron en la investigación, en general declaran tener una percepción positiva de la formación recibida en la escuela. Consideran que fue buena, que les permitió explorar diferentes áreas de la arquitectura y explorar sus ideas (EG-4M-5) o que la formación real como arquitecto se inició en su 5to año cuando pudieron escoger su área de especialización (EP-F-8). Incluso algunos mencionan que los talleres anteriores tienen gran peso, en especial talleres 9 y 10 , que llegan a ser de los más completos al poseer el apoyo de los ramos de investigación, lo cual consideran que, en conjunto, llega a veces a superar la complejidad del proceso de titulación (EP-M-10).

Los sujetos de estudio están altamente conscientes de las competencias que como profesionales requieren (EP 95\% - EG -83\%). Sin embargo, no se sienten preparados para enfrentar el mundo profesional. (EP 63\% - EG 80\%).

En cuanto al proceso de titulación, las opiniones positivas disminuyen, aun así hay quienes destacan que permite profundizar en desarrollo del proyecto y resolver de manera más integral las aristas del mismo (EG-3F-8), que obliga a madurar en cuanto a las metodologías de trabajo (EP-F-11), permite desarrollar investigaciones propias que podrían llegar a transformarse en una posibilidad de nicho laboral (EG-5M-4) o simplemente lo rescatan porque será el proyecto más completo que tendrán para mostrar en el futuro. A pesar de esto, la mayoría señala que no se asemeja al ejercicio profesional (EP 92\% - EG 85\%).

Los estudiantes declaran que el actual proceso de titulación busca que ellos cumplan con los parámetros establecidos tradicionalmente para completar su presentación, y esto no necesariamente estaría vinculado con el mundo laboral (EP 75\%- EG 80\%), además consideran que gran parte del proceso y del resultado final se pierden en el actual formato (EP-M-10).

Las instituciones parecieran considerar que este proceso promueve competencias valiosas para actividades diversas, pero lo cierto es que está basado en un modelo concreto de ejercicio profesional que al parecer no constituye la práctica habitual de la mayoría de los arquitectos (Puig, 2017). Es más, se estima que actualmente el $75 \%$ de los arquitectos se están desempeñando en otros campos diferentes a los tradicionales de la profesión (González-Couret, 2014).

La percepción positiva o negativa de los egresados en el futuro dependerá en gran 
medida de la dificultad que tengan para incorporarse al mercado laboral. Esta inserción, por otra parte, depende de si las competencias obtenidas durante su paso en la universidad son coincidentes con lo que los empleadores están requiriendo (González-Jaimes, 2016) y podemos inferir a través de lo expresado tanto por estudiantes y egresados que es ahí donde se puede evidenciar la brecha que existe entre academia y ejercicio profesional. Declaran que los conocimientos que son relevantes en su ejercicio profesional, no provienen de la universidad, sino que fueron adquiridos desde el momento en que salieron de la escuela (EG-3M-20).

Los arquitectos necesitan, más que nunca, altas dosis de inspiración que les permitan identificar o inventar nuevas fórmulas de ejercicio profesional, sin renunciar al rol que han jugado hasta ahora. (Puig, 2017). Aprender a ser arquitecto, en definitiva, consiste en ser un experto del diseño, es decir, aportar soluciones concretas a problemas más o menos genéricos e indefinidos. Es por ello que, en el trabajo del arquitecto, y por ende en el aprendizaje del estudiante de arquitectura, la creatividad y la innovación son consustanciales, son la materia central de los talleres de arquitectura (SolaMorales, 2016). A pesar de esto, vemos que la forma de poner a prueba la formación recibida se mantiene sin cambios significativos por décadas. Podemos afirmar de esta manera que la escuela considera que el proyecto de título es la finalización de la formación académica, a pesar de que diferentes agentes de la sociedad están exigiendo que esta sea la primera de la etapa profesional del estudiante.

Los empleadores por una parte exigen competencias que la universidad no entrega, (Gonzalez-jaimes, 2016) y, por ende, no son puestas a prueba en este proceso. Por otra parte, los estudiantes exigen que el proceso recoja sus inquietudes y talentos, los que podrán convertirse en proyectos reales, redes de contacto, trabajos con agentes comunitarios e incluso mencionan la posibilidad de realizar prácticas guiadas (EP-F-8). Sin embargo, los seguimos poniendo en situaciones que en la realidad laboral no existen: trabajar absolutamente solos, sin presupuesto, no coordinar instalaciones, no aplicar conocimientos de normativa y tramitación municipal, etc. (EG-4F-6). Debemos considerar que hoy en día, las prácticas tradicionales de la profesión no pueden considerarse suficientes ni todas ellas adecuadas para los todos los arquitectos contemporáneos. (Puig, 2017). No es posible hoy homologar o seguir cifrando el ejercicio de la profesión exclusivamente en el diseño de edificios. Si consideramos el contexto de crisis económica actual, la adquisición de una titulación universitaria adquiere un significado importante para hacer frente a la vida laboral y evitar situaciones de precariedad a futuro. (Sanchez-Gelabert, 2016). Nuestros egresados no consideran su proyecto de título como una herramienta a la hora de buscar trabajo a pesar de todo el esfuerzo, tiempo y dinero invertido en ello (EP 100\% - EG 85\%). Todo esto supone un nuevo impulso para afrontar las crecientes dificultades relacionadas con la inserción laboral de los titulados y los desajustes entre la formación recibida y los requisitos de los puestos de trabajo. (Michavila, 
2018). ¿Qué factores deben mejorar para que sus egresados cumplan con las demandas del campo laboral? ¿Qué áreas profesionales requiere el mercado laboral a mediano y largo plazo? (González-Jaimes, 2016). Responder estas preguntas resulta clave para la reformulación del proceso en busca de generar un aporte a la formación profesional e inserción laboral de nuestros egresados.

\section{Conclusiones y discusión}

Los datos levantados indican que los estudiantes finalizan su carrera con sentimientos encontrados, por una parte, tienen la idea de que su proyecto de título será un aporte para la sociedad, que abordan temas contingentes a la disciplina y que al finalizar su proceso de titulación se encontrarán capacitados para el mercado laboral. Al mismo tiempo y en gran medida, después de pasar por distintos programas de prácticas, consideran que su proyecto de título no será la herramienta con la cual iniciarán la búsqueda laboral, están plenamente conscientes de la brecha que existe entre la academia y el mundo real y del mismo modo, entienden que el proceso de titulación no es similar al ejercicio profesional y que el campo laboral del arquitecto no se limita al diseño de edificios.

Por otra parte, la gran mayoría de los egresados sienten que su proyecto de título no es relevante para su desempeño profesional, menos de la mitad cree que haya sido un aporte para la sociedad y aunque declaran que el proyecto no fue clave en su búsqueda laboral, ni les aseguró el puesto en el que se desempeñan, tienen absoluta certeza de que el ejercicio profesional no es similar al desarrollado en el proceso de titulación. Al mismo tiempo, declaran que en este proceso sí utilizaron todas sus competencias profesionales, que la formación de la universidad es buena y que valoran la investigación desarrollada y quisieran, en muchos casos, que se potenciará el trabajo de tesis, las relaciones con organismos públicos y/o privados y la creación de redes y contactos. Consideran que la universidad debería buscar aportar en la creación de redes y de esta manera abrir nuevas opciones de titulación, ya que no todos somos iguales y no a todos nos gusta hacer lo mismo. En síntesis, el campo laboral actual del arquitecto exige a la universidad severos cambios y actualizaciones.

La mayoría de los jóvenes profesionales, cuando observan de manera retrospectiva su proceso de titulación, tienen una percepción negativa. La observación más común entre ellos es que el proceso y/o el proyecto de titulación no les generó ningún aporte, que no les ayudó ni facilitó el ingreso al mercado laboral. Distinguen la gran distancia que existe entre la academia y el mundo real, calificándola incluso como una utopía. 
Paralelamente, destacan que el programa de prácticas fue clave en su formación y que los 6 años (o más) de universidad los prepararon en términos generales para su formación como profesional.

Los estudiantes ven su actual proceso de titulación con gran incertidumbre, frustración e incluso molestia respecto a su futuro laboral. La incertidumbre se origina principalmente porque no ven que el proceso de titulación genere algún aporte o que los prepare para el mercado laboral. Las casi 500 horas de prácticas que poseen a lo largo de la carrera les han permitido ver esto, incluso estudiantes en proceso hablan de la universidad como una ilusión que promete ser algo que en realidad no es. Estos mismos estudiantes ven compañeros que se han titulado años anteriores con nota máxima y que se encuentran desempleados. Luego perciben que de parte de la escuela esta etapa se considera un mero trámite, no sienten en su mayoría un respaldo de la institución o de los profesores involucrados. Los estudiantes en proceso que participaron en la investigación aportaron numerosas ideas de cómo se podría mejorar, recogidas de las experiencias obtenidas en intercambios o diálogos con estudiantes de otras universidades chilenas y extranjeras. Consideran que el título es la finalización de una etapa académica de la vida, que los talleres previos los prepararon para eso, que les permite profundizar en sus conocimientos y que se ponen a prueba a sí mismos en cuanto a manejar niveles de frustración altísimos, gran cantidad de trabajo, periodos excesivamente largos de trabajo, desconexión con el mundo real y al mismo tiempo, declaran que todo esto ya lo vivieron el año anterior y que incluso del taller previo al de título era más duro, pero que contaban con el apoyo y participación de más profesores en el proceso, lo que lo hacía más consistente. De esta manera, podemos apreciar que el proceso de título para algunos es redundante.

El proceso de titulación basado únicamente en un proyecto ficticio, responde a la finalización de un modelo académico que tiene como pilar al docente y a la enseñanza. La universidad en estudio hace algunos años cambió esto al adscribirse a un enfoque basado en competencias. De esta manera, el foco de esta escuela está en el estudiante y en su aprendizaje, con esto se ha re articulado el modelo y se ha actualizado el plan de estudios. Durante la carrera se observa un estudiante mejor preparado, que entiende las problemáticas del mercado, conoce lo que éste le exige y no tiene problemas para sobrevivir en él. Sin embargo, la manera en que se ponen a prueba las competencias profesionales que esta institución les ha ayudado a formar sigue siendo la misma. Hoy el hito más importante en la conclusión de su formación universitaria se encuentra disociado tanto de la formación entregada como del mercado laboral próximo. 
La sociedad y el mercado laboral han cambiado con constante celeridad durante los últimos años, los recién egresados son más sensibles a ello, coincidentemente a esto existen cada vez más escuelas de arquitectura que están abriendo el abanico de posibilidades de titulación buscando por una parte recoger los intereses personales de sus egresados y por otra, facilitarle el ingreso al cada vez más competitivo mercado laboral.

Al comparar las diferentes opiniones de los dos grupos participantes, encontramos un gran número de coincidencias. Éstas guardan relación al poco o nulo aporte del proceso de titulación para su inserción laboral, lo redundante del último año y el proceso de título y la actual brecha entre la academia y el ejercicio profesional. Ambos grupos coinciden en destacar el aporte el programa de prácticas y de la formación global de su paso por la universidad, los 5 años (10 talleres) que pusieron a prueba las competencias adquiridas en la totalidad de asignaturas.

Podemos ver también cómo las expectativas de que el proyecto de título sea relevante tanto en su campo profesional y/o sociedad no se concretan en más de la mitad de los casos. Los estudiantes son mucho más críticos del proceso actual en cuanto a su orden, formulación y apoyo de la institución que los egresados.

\section{Referencias}

Alba-Dorado, M., Evaluación formativa y compartida en el aprendizaje de la Arquitectura. En Garcia, D; Bardí, B. y Domingo, D. (eds.) (2016) IV Jornadas sobre Innovación Docente en Arquitectura (JIDA'16), Escuela Técnica Superior de Arquitectura de Valencia, 20 y 21 de octubre de 2016. UPV; UPC. http://dx.doi.org/10.5821/jida.2016.5093

Cruz, M. (2018). La formación del arquitecto como agente de cambio en la ciudad del siglo XXI. Arquitectura y Urbanismo, 39(2), 109-116. http://rau.cujae.edu.cu/index.php/revistaau/article/view/497

Fox, H. (2017). Reflexiones en torno al proceso de diseño en arquitectura. AUS [Arquitectura / Urbanismo / Sustentabilidad], (5), 4-9. http://revistas.uach.cl/index.php/aus/article/view/643

González, E. y Miguel, M. (2017). Egresados universitarios y su pertinencia para el ingreso a la empleabilidad empresarial o la auto-empleabilidad. Revista Electrónica de psicología Iztacala, 20(1), 168-185. 
González, D. (2014). Innovación y diversidad: Retos y oportunidades en Arquitectura, Diseño y Urbanismo. Reseña de la Segunda Conferencia ALFA ADU_2020. Arquitectura $\quad y \quad$ Urbanismo, 35(1), 104-110. http://scielo.sld.cu/scielo.php?script=sci_arttext\&pid=S181558982014000100010\&lng=es\&tIng=es

González, H., y Sánchez, T. (2016). Saber pedagógico: fundamento del ejercicio docente. Educación y Educadores, 19(2), 241-253. http://dx.doi.org/10.5294/edu.2016.19.2.4

González-Jaimes, E., (2016). Competencias académicas de los egresados universitarios y su predicción de ocupación laboral. Revista lberoamericana de las Ciencias Sociales y Humanísticas, 5(10). http://www.redalyc.org/articulo.oa?id=503954317004

Maragaño, A. (2017). Las ligeras formas del habitar. Algunas partes del modelo educativo de la escuela de arquitectura de la Universidad de Talca. Revista Europea de Investigación en Arquitectura, 07-08, 190-197. http://hdl.handle.net/11268/6278

Matamoros, M., Gutiérrez, R., Rouco, A. y Collado, N. (2015). Diseño, investigación y formación. Experiencias en los trabajos de diploma. Arquitectura $y$ Urbanismo, 36(1), 116-125. http://scielo.sld.cu/scielo.php?script=sci_arttext\&pid=S181558982015000100011\&lng=es\&tlng=es.

Michavila, F., Martinez, J., Martín-González, M., García-Peñalvo, F. y Cruz-Benito, J. (2018).

Empleabilidad de los titulados universitarios en España. Proyecto OEEU. Education in the Knowledge Society (EKS), 19(1), 21-39. http://dx.doi.org/10.14201/eks20181912139

Muñoz, C., Morales, J., Riveros, J., Parada, M., Ibarra, J. y Ramírez, L., (2015). Aprendizaje y servicio en el proyecto de título, una propuesta de innovación docente en Arquitectura. Arquitectura y Cultura, 26, 86-98.

Puig-Pey, A. (2017). El arquitecto: formación, competencias y práctica profesional. Architecture, City and Environment. 12(34), 301-320. http://dx.doi.org/10.5821/ace.12.34.5296

Quesada, M., Leyva, C. y Menchero, M. (2016). La formación de habilidades profesionales del arquitecto. Transformación. 12(3), 403-414. 
Rodríguez, A., (2014). Los desafíos del Taller Terminal de Arquitectura. Desarrollando los fundamentos del futuro arquitecto. Arquitectura y Urbanismo, 35(3), 121129.

http://scielo.sld.cu/scielo.php?script=sci_arttext\&pid=S1815$58982014000300010 \& \operatorname{lng}=e s \&$ tlng=es

Rodríguez, Y. (2017). Reconceptualización de la educación en la era digital.. Revista De $\begin{array}{lllll}\text { Comunicación } & \text { De } & \text { La S5-118. }\end{array}$ http://dx.doi.org/10.15198/seeci.2017.42.85-118

Sanchez-Gelabert, A., Navarro-Cendejas, J. (2016). La valoración que los graduados tienen sobre su formación universitaria desde su situación laboral actual. Revista Complutense De Educación, 27(2), 669-688. http://dx.doi.org/10.5209/rev_RCED.2016.v27.n2.47007

Sola-Morales, P., Ávila-Martorell, G., García, M., Rabassa, N. y Romeu, M. (2016). Definición de la competencia transversal sobre "creatividad, innovación y resolución de problemas. IV Jornadas sobre Innovación Docente en Arquitectura (JIDA'16), Escuela Técnica Superior de Arquitectura de Valencia, 20 y 21 de octubre de 2016. UPV;UPC. http://dx.doi.org/10.5821/jida.2016.5129 


\begin{abstract}
Domingo Sarmiento L.
Arquitecto Universidad Mayor, Magíster en Docencia para la Educación Superior, Universidad Central, forma parte del primer registro de pares evaluadores en la Comisión Nacional de Acreditación CNA. Presidente del Comité de Arquitectos Jóvenes, del Colegio de Arquitectos de Chile, entre los años 2017 y 2020, actualmente integra el comité de docencia del Colegio de Arquitectos de Chile. Profesor universitario en la escuela de arquitectura de la universidad Mayor entre los años 2009 y 2016, actualmente es profesor de taller en la escuela de Arquitectura de la Universidad Central, y profesor Asociado en las escuelas de Arquitectura y Diseño Interior de la Universidad UNIACC. Hoy en día, mantiene su labor docente en paralelo al ejercicio profesional, siendo Socio de la oficina Sarmiento Zamora Arquitectos Asociados SZAA, con obras proyectadas a lo largo de todo el país.

Teléfono: +56930570641

Email: domingo.sarmiento@uniacc.edu
\end{abstract}

\title{
Guillermo Caro P.
}

Arquitecto Universidad Mayor, Magíster en Docencia para la Educación Superior Universidad Central de Chile, Diplomado en Calidad Educativa de la Educación Superior, Diplomado en Investigación Universitaria, Diplomado en Docencia para la Educación Superior, Investigador colaborador del Grupo Independiente de Investigación Educativa, proyecto “Creatividad: Competencia Oculta”, expuesto en el XXI congreso Internacional de Aprendizaje y Enseñanza, Nueva York 2014, Investigador principal del proyecto "Formación del Arquitecto: La incidencia del proyecto del proyecto de título en el campo laboral" expuesto en el XII congreso internacional de Investigadores en Educación , Osorno 2019. Coordinador durante 2 años del Sistema de Portafolios para el proceso de Acreditación Internacional RIBA 2019 de la Escuela de Arquitectura de la universidad Central de Chile, con 10 años de experiencia docente enfocados en el desarrollo de la creatividad proyectual destacando principalmente: Talleres de Arquitectura, Talleres de creación audiovisual, Formación de docentes en utilización TICS para el aula, actualmente profesor asociado en al Facultad de Arquitectura, Diseño y Artes de la Universidad UNIACC. Secretario del Comité de Docencia del Colegio de Arquitectos de Chile. Profesionalmente colaborando en diferentes oficinas tales como ENLACE arquitectura y gestión, EMESE arquitectura, principalmente en proyectos como restaurantes, oficinas, institutos Teletón, arquitectura educacional, entre otros.

Teléfono: +56991266483

Email: guillermow.caro@uniacc.edu 